\title{
A Pediatric Covariate Function for CYP3A-Mediated Midazolam Clearance Can Scale Clearance of Selected CYP3A Substrates in Children
}

\author{
Janneke M. Brussee, ${ }^{1}$ (D) Elke H. J. Krekels, ${ }^{1}$ Elisa A. M. Calvier, ${ }^{1}$ Semra Palić, ${ }^{2}$ Amin Rostami-Hodjegan, ${ }^{3,4}$ \\ Meindert Danhof, ${ }^{1}$ Jeffrey S. Barrett, ${ }^{5,6}$ Saskia N. de Wildt, ${ }^{7,8}$ and Catherijne A. J. Knibbe ${ }^{1,9,10}$
}

Received 1 March 2019; accepted 7 June 2019; published online 27 June 2019

\begin{abstract}
Recently a framework was presented to assess whether pediatric covariate models for clearance can be extrapolated between drugs sharing elimination pathways, based on extraction ratio, protein binding, and other drug properties. Here we evaluate when a pediatric covariate function for midazolam clearance can be used to scale clearance of other CYP3A substrates. A population PK model including a covariate function for clearance was developed for midazolam in children aged 117 years. Commonly used CYP3A substrates were selected and using the framework, it was assessed whether the midazolam covariate function accurately scales their clearance. For eight substrates, reported pediatric clearance values were compared numerically and graphically with clearance values scaled using the midazolam covariate function. For sildenafil, clearance values obtained with population PK modeling based on pediatric concentration-time data were compared with those scaled with the midazolam covariate function. According to the framework, a midazolam covariate function will lead to systemically accurate clearance scaling (absolute prediction error $(\mathrm{PE})<30 \%$ ) for CYP3A substrates binding to albumin with an extraction ratio between 0.35 and 0.65 when binding $<10 \%$ in adults, between 0.05 and 0.55 when binding $>90 \%$, and with an extraction ratio ranging between these values when binding between 10 and $90 \%$. Scaled clearance values for eight commonly used CYP3A substrates were reasonably accurate $(\mathrm{PE}<50 \%)$. Scaling of sildenafil clearance was accurate $(\mathrm{PE}<30 \%)$. We defined for which CYP3A substrates a pediatric covariate function for midazolam clearance can accurately scale plasma clearance in children. This scaling approach may be useful for CYP3A substrates with scarce or no available pediatric PK information.
\end{abstract}

KEY WORDS: Clearance; CYP3A ontogeny; Scaling function; Pediatrics; Population pharmacokinetics.

Electronic supplementary material The online version of this article (https://doi.org/10.1208/s12248-019-0351-9) contains supplementary material, which is available to authorized users.

\footnotetext{
${ }^{1}$ Division of Systems Biomedicine and Pharmacology, Leiden Academic Centre for Drug Research (LACDR), Leiden University, Leiden, The Netherlands.

${ }^{2}$ Present Address: Dutch Cancer Institute (NKI), Amsterdam, The Netherlands.

${ }^{3}$ Centre for Applied Pharmacokinetic Research, University of Manchester, Manchester, UK.

${ }^{4}$ Simcyp Limited (A Certara Company), Sheffield, UK.

${ }^{5}$ Bill \& Melinda Gates Medical Research Institute, Cambridge, Massachusetts, USA.

${ }^{6}$ Department of Pediatrics, Division of Clinical Pharmacology \& Therapeutics, Children's Hospital of Philadelphia, Philadelphia, Pennsylvania, USA.

${ }^{7}$ Department of Pharmacology and Toxicology, Radboud University Medical Centre, Nijmegen, The Netherlands.

${ }^{8}$ Intensive Care and Department of Pediatric Surgery, Erasmus MC Sophia Children's Hospital, Rotterdam, The Netherlands.

${ }_{9}^{9}$ Department of Clinical Pharmacy, St. Antonius Hospital, PO Box 2500, 3430, EMNieuwegein, The Netherlands.

${ }^{10}$ To whom correspondence should be addressed. (e-mail: c.knibbe@antoniusziekenhuis.nl)
} 


\section{INTRODUCTION}

To define the optimal first-in-child dose during drug development and to develop pediatric dose recommendations for clinical practice, accurate scaling of the plasma clearance of drugs is essential (1-3). This is of particular relevance as performing dedicated pharmacokinetic (PK) studies for all drugs in all pediatric (sub)populations may not be feasible because it would take too many resources. Moreover, this may not even be necessary when other methods are available and could therefore even be considered unethical. One proposed approach shares PK information of drugs eliminated by the same pathway by extrapolating covariate relationships for clearance between drugs (4). This has already been successfully applied for scaling pediatric clearance for drugs glucuronidated by UGT2B7 enzymes and also for drugs eliminated by glomerular filtration (4-6).

Within this context, recently, a framework was presented by Calvier et al. for hepatically metabolized drugs identifying the conditions for which between-drug extrapolation is systematically accurate (7). This framework takes into account changes in physiological parameters with age, including changes in (hepatic) blood flow, plasma protein concentrations, hematocrit, liver size, the amount of microsomal protein per gram of liver, and the ontogeny of isoenzyme expression (the microsomal intrinsic clearance) (7). One of the key findings of this framework was that the accuracy of this scaling method depends on the fraction metabolized by the isoenzyme pathway for which plasma clearance is scaled, on the hepatic extraction ratio of both the probe drug and the evaluated drugs in adults, on the type of binding plasma protein, and on the unbound drug fraction $\left(f_{\mathrm{u}}\right)$ in adults $(7)$.

As many drugs are eliminated by the cytochrome P450 (CYP) 3A enzyme family $(8,9)$, a pediatric covariate function for CYP3A-mediated clearance may aid in scaling clearance of CYP3A substrates. Midazolam is an established probe drug for CYP3A-mediated clearance $(10,11)$, mainly metabolized by CYP3A4, and to a lesser extent by CYP3A5 (12), and has an intermediate extraction ratio (13). Our aim is to evaluate when a pediatric covariate function for midazolam clearance can be used to scale clearance of other CYP3A substrates in children, taking into account the recent insights of the developed framework (7).

\section{METHODS}

\section{Overall Approach}

A population PK model for midazolam in children was developed based on concentration-time data, to establish a pediatric covariate function for midazolam clearance. Next, we selected a range of drugs that are CYP3A substrates that are commonly prescribed in children, covering compounds prescribed for varying indications in different therapeutic areas, with oral or intravenous administration, and with different drug properties, i.e., alprazolam (14), atorvastatin (15), cisapride (16), domperidone (17), quinidine (18), sildenafil (19), simvastatin (20), solifenacin (21), sufentanil (22), sirolimus (23), tacrolimus (24), tamsulosin (25), and vincristine (26). Based on the drug properties of these CYP3A substrates, we used the framework of Calvier et al. (7) to define which age the covariate function for midazolam can be used for accurate scaling of pediatric clearance of the CYP3A substrates from adult clearance values. For eight of the selected CYP3A substrates, pediatric and adult clearance values were available in literature, allowing for the assessment of the accuracy of the scaling function by comparing pediatric clearance values that were scaled from adult clearance values using the covariate function for midazolam to the published literature clearance values in children. Furthermore, for sildenafil, concentration-time data were available from 156 children (27). Using these data, we developed two pediatric population PK models for sildenafil; one using the pediatric covariate function of midazolam clearance directly and one in which the covariate relationship for clearance was optimized using a data-driven analysis, after which, the performance of both models, as well as the estimated and scaled clearance values, was compared.

\section{Midazolam Population PK Model}

Midazolam PK data were available from 31 patients (15 male, 16 female) from the Children's Hospital of Philadelphia, PA (Table I), with a median age of 8 years of age (range 1-17 years) and a median body weight of $30.2 \mathrm{~kg}$ (range 9.5$83.2 \mathrm{~kg}$ ) (28). Before participation, signed informed consent, by the subject's parents or guardian, and assent were obtained. Children undergoing surgery were included if they met the criteria I or II of the American Society of Anesthesiologist's (ASA) classification. A median dose of $12.5 \mathrm{mg}$ (range 3-15 mg) of midazolam was administered as oral suspension $(5 \mathrm{mg} / \mathrm{mL}$, Roche

Table I. Study and Patient Characteristics of the Midazolam and Sildenafil PK Studies

\begin{tabular}{lll}
\hline & Midazolam & Sildenafil \\
\hline Indication & Pre-operatively & Pulmonary arterial hypertension \\
Number of patients & 31 & 156 \\
Number of samples & 327 & 591 \\
Samples/patient* & $10(8-11)$ & $4(1-4)$ \\
Age (years)* & $8(1-17)$ & $10(1-17)$ \\
Body weight $(\mathrm{kg}) *$ & $30.2(9.5-83.2)$ & $28.0(8.2-106.0)$ \\
Male/female, $n(\%)$ & $15 / 16(48 / 52 \%)$ & $57 / 99(37 / 63 \%)$ \\
Dose $(\mathrm{mg})^{*}$ & $12.5(3-15)$ & $20(10-80)$ \\
\hline
\end{tabular}

*median (range) 
Laboratories) to the patients pre-operatively. Blood was densely sampled for midazolam plasma concentrations around $0.25,0.5,1$, $1.5,2,3,4,6,8,10$, and $22 \mathrm{~h}$ after dose administration, with a median of 10 samples per patient (range 8-11). Blood was centrifuged and plasma samples stored at $<-20{ }^{\circ} \mathrm{C}$, until midazolam plasma concentrations were determined using LC/MS (28).

A population $\mathrm{PK}$ model was developed using non-linear mixed effects modeling (NONMEM version 7.3, ICON, Globomax LLC, Ellicott, MD, USA; Perl-speaks-NONMEM (PsN) version 4.2.0, Uppsala, Sweden; and Pirana 2.9.0, Pirana Software \& Consulting BV, Denekamp, the Netherlands) based on first-order conditional estimation with interaction. R (version 3.3.1) and RStudio (version 0.98.1078) were used for data visualization. Several structural models were considered, including 1-, 2-, and 3-compartmental models, and evaluated based on criteria for model stability, goodness-of-fit, and parameter precision, and on comparisons of the objective function values (OFV, $-2 \times \log$-likelihood), using a significance level of $p<0.05$. The absorption rate could not be estimated and was therefore fixed at $3.5 \mathrm{~h}^{-1}(29)$, which results in $\mathrm{t} t_{\max }$ around $0.5 \mathrm{~h}$ postdose, which is in agreement with known values.

Interindividual variability in the estimated parameters for clearance and central volume of distribution was included in the model by the following equation:

$P_{i}=\theta_{\mathrm{TV}} \times e^{\eta_{i}}$

in which $P_{i}$ is the individual parameter estimate for individual $i, \theta_{\mathrm{TV}}$ is the typical value of the parameter in the studied population, and $\eta_{i}$ is a random variable for the $i$ th individual from a normal distribution with a mean of zero and variance of $\omega^{2}$, assuming a log-normal distribution for the parameter value in the population.

To describe residual unexplained variability, a proportional error model, an additive error model, and a combination of the proportional and additive error were considered. The $j$ th observed concentration of the $i$ th individual $\left(Y_{i j}\right)$ was modeled according to

$Y_{i j}=C_{\text {pred }, i j} \times\left(1+\varepsilon 1_{i j}\right)+\varepsilon 2_{i j}$

where $C_{\mathrm{pred}, i j}$ is the $j$ th predicted midazolam concentration of the $i$ th individual, and $\varepsilon_{i j}$ is a random variable from a normal distribution with a mean of zero and variance of $\sigma^{2}$, with $\varepsilon 1$ the proportional error and $\varepsilon 2$ the additive error.

A systematic covariate analysis was performed for the estimated model parameters in which age, body weight, and sex were tested for statistical significance. For sex, the typical value $\left(\theta_{\mathrm{TV}}\right)$ for girls was estimated relative to the value for boys. The remaining continuous covariates body weight and age were tested using a power (Eq. 3) function:

$P_{i}=\theta_{\mathrm{TV}} \times\left(\frac{\mathrm{COV}_{i}}{\mathrm{COVmed}}\right)^{\theta_{\mathrm{Cov}}} \times e^{\eta_{i}}$

where $P_{i}$ is the individual parameter estimate for individual $i$ with a covariate value of $\mathrm{COV}_{i}, \theta_{\mathrm{TV}}$ is the parameter value for a typical individual with a median covariate value $\left(\mathrm{COV}_{\mathrm{med}}\right)$, $\theta_{\mathrm{COV}}$ is the estimated exponent, and $\eta_{i}$ is a random variable as described above (Eq. 1). For the forward inclusion of a covariate, a drop in OFV by at least 6.64 points $(p<0.01)$ was considered statistically significant, while for the backward deletion a more stringent $p$ value $(p<0.005, \Delta \mathrm{OFV}>7.88)$ was used. In addition, the interindividual variability in the PK parameter or the residual variability should decrease for a covariate to be retained in the model.

To evaluate whether the model described the observed concentrations well, goodness-of-fit plots were assessed. These diagnostic plots include observed versus populationand individual-predicted concentrations and conditional weighted residuals (CWRES) versus population-predicted concentrations and versus time. To evaluate model stability and parameter precision, a bootstrap analysis $(n=250)$ was performed. Finally, a normalized prediction distribution error (NPDE) analysis was performed using the NPDE package in $\mathrm{R}$ (30), with $n=1000$ simulations to evaluate whether the model can accurately predict the concentration and capture the observed variability.

\section{Between-Drug Extrapolation Potential of Midazolam Clearance to Other CYP3A Substrates}

The previously published framework on between-drug extrapolation of covariate functions (7) was used to assess, based on the drug properties of CYP3A substrates, whether between-drug extrapolation of the covariate relationship for midazolam would lead to accurate scaling of the pediatric clearance of the selected CYP3A substrates. For this, the relevant drug properties, i.e., the extraction ratio, the plasma protein to which the drug is binding, and the $f_{\mathrm{u}}$ for midazolam and the selected drugs were obtained from literature. In this analysis, the selected drugs were assumed to exclusively bind to either human serum albumin (HSA) or $\alpha 1$-acid glycoprotein (AAG), while midazolam was assumed to bind to either HSA (for comparison with HSA-binding drugs) or to AAG (for comparison with AAG-binding drugs).

Using the extraction ratio and the $f_{\mathrm{u}}$ of the selected CYP3A substrates that were considered within the results from the framework (7), it was assessed to what age clearance scaling with the covariate function of midazolam would certainly be accurate for the selected drugs. Drugs were selected of which it has been reported that CYP3A is the "major" pathway for elimination, and we assumed CYP3A metabolism to be responsible for $\geq 75 \%$ of the total metabolism for both midazolam and all selected substrates. Based on the extraction ratio and $f_{\mathrm{u}}$ from midazolam, we also derived general criteria for systematically accurate clearance scaling for CYP3A substrates using the covariate function for midazolam clearance according to the framework.

\section{Comparison of Scaled Versus Reported Pediatric Clearance Values}

For the selected CYP3A substrates for which both pediatric and adult clearance values were reported in literature, we applied the pediatric covariate function for midazolam clearance to the reported adult clearance values to scale for pediatric clearance values. For this we assumed that typical adults have a body weight of $70 \mathrm{~kg}$. We graphically compared the scaled typical clearance values with the reported pediatric clearance values. Moreover, we calculated the prediction error (PE) for three typical subjects (an infant of $10 \mathrm{~kg}$, a child of $20 \mathrm{~kg}$, and an adolescent of $50 \mathrm{~kg}$ ) based on literature values for pediatric clearance using Eq. 4: 
$\mathrm{PE}=\frac{\mathrm{CL}_{\mathrm{scaled}}-\mathrm{CL}_{\mathrm{ref}}}{\mathrm{CL}_{\mathrm{ref}}} \times 100 \%$

with $\mathrm{CL}_{\text {scaled }}$ the scaled clearance value and $\mathrm{CL}_{\text {ref }}$ the reported pediatric clearance. An absolute $\mathrm{PE}$ of $<30 \%$ was considered accurate, an absolute PE of 30-50\% reasonably accurate, and an absolute $\mathrm{PE}$ of $\geq 50 \%$ inaccurate.

\section{Sildenafil Population PK Models}

Sildenafil PK data from a previously published study (27) were made available by Pfizer Inc. In this study, sildenafil PK data were collected from 156 (57 male, 99 female) patients in a randomized, double-blind, placebo-controlled, dose ranging, parallel group study of oral sildenafil for the treatment of children with pulmonary arterial hypertension $(27,31)$. Subjects included children ranging in age from 1 to 17 years (median 10 years), with a median body weight of $28.0 \mathrm{~kg}$ (range 8.2-106 kg) (Table I). A median of four samples per patient (range 1-4) was available, with a total of 591 measurements available for analysis. Samples were taken at steady-state at trough and around three, six, and eight hours postdose. Patients were randomly assigned to a low-, medium-, or highdose group ( $n=39, n=48$, and $n=69$, respectively), and the dosages were weight-stratified, with a medium dose of 10,20 , and $40 \mathrm{mg}$ and a high dose of 20,40 , and $80 \mathrm{mg}$ for patients of $8-20 \mathrm{~kg}$, $20-45 \mathrm{~kg}$ or $>45 \mathrm{~kg}$, respectively. The low dose was $10 \mathrm{mg}$ for all patients $>20 \mathrm{~kg}$, and patients with a body weight $\leq 20 \mathrm{~kg}$ received either a medium or high dose, as no drug effect was expected with a lower dose than $10 \mathrm{mg}(27,31)$. In the population PK analysis, the samples without recorded sampling times were excluded.

Based on these data, a "reference model" was developed in the same manner as described for midazolam. The absorption rate constant could not be estimated and was therefore fixed at $1 \mathrm{~h}^{-1}$, leading to a maximum concentration around two hours post-dose, which was before the first sample was taken.

The extrapolation potential of the covariate function for midazolam clearance was evaluated in a second population PK model referred to as the "extrapolation model." This model was kept the same as the reference model, except the clearance was not estimated, but scaled from an apparent CL/F value of $100 \mathrm{~L} / \mathrm{h}$ for adults, which was derived from reported systemic clearance and oral bioavailability values of $41 \mathrm{~L} / \mathrm{h}$ and $0.41 \mathrm{~L} / \mathrm{h}$, respectively (32), using the covariate function for midazolam clearance. We assumed the same bioavailability in adults and pediatric patients.

The reference and extrapolation models were evaluated in the same manner as the midazolam PK model (see under "Midazolam Population PK Model").

Sildenafil clearance values from the sildenafil the "reference model" $\left(\mathrm{CL}_{\mathrm{ref}}\right)$ and the sildenafil "extrapolation model" $\left(\mathrm{CL}_{\text {scaled }}\right)$ were compared graphically. For a numerical comparison of both sildenafil models, typical clearance values for three typical subjects (an infant of $10 \mathrm{~kg}$, a child of $20 \mathrm{~kg}$, and an adolescent of $50 \mathrm{~kg}$ ) were calculated, and a PE for clearance was calculated using Eq. 4.

\section{RESULTS}

\section{Midazolam Population PK Model}

For midazolam, a two-compartmental model with body weight, included in an exponential covariate relationship on clearance, volumes of distribution, and intercompartmental clearance, best described the data (Table II, fig. S2, S3). As midazolam was administered orally, apparent parameters for clearance and volume of distribution were obtained. For a typical individual of $30.2 \mathrm{~kg}$, apparent clearance was $102.6 \mathrm{~L} / \mathrm{h}$, and the exponent, in the exponential equation relating body weight and clearance, was found to be 0.874 (Table II). As a result, this pediatric covariate function was used to scale CYP3A-mediated clearance in the between-drug extrapolation:

$\mathrm{CL}_{\text {pediatric }}=\mathrm{CL}_{\text {adult }} \times\left(\frac{\mathrm{WT}}{70}\right)^{0.874}$

\section{Between-Drug Extrapolation Potential of Midazolam Clearance to Other CYP3A Substrates}

The obtained drug properties of midazolam and the selected CYP3A substrates required for between-drug extrapolation of clearance are listed in Table SI (33-54). Figure S1 shows down to

Table II. Model Parameter Estimates for the Midazolam PK Model and the Bootstrap Results Based on $n=250$ Resampling

\begin{tabular}{llll}
\hline Parameter & & Model estimate (RSE) & Bootstrap median (90 CI) \\
\hline Midazolam clearance $^{\dagger}$ & $(\mathrm{CL} / \mathrm{F})_{30.2 \mathrm{~kg}}(\mathrm{~L} / \mathrm{h})$ & $102.6(9 \%)$ & $101.4(89.1-118.2)$ \\
$\mathrm{CL}_{i}=\mathrm{CL}_{30.2 \mathrm{~kg}} \times\left(\mathrm{WT}_{i} / 30.2\right)^{\mathrm{k} 1}$ & $k 1$ & $0.874(13 \%)$ & $0.901(0.698-1.11)$ \\
Volume of distribution $^{\dagger}$ & $\left(\mathrm{V}_{\mathrm{c}} / \mathrm{F}\right)_{30.2 \mathrm{~kg}}(\mathrm{~L})$ & $156(25 \%)$ & $141(76.5-210)$ \\
$V_{\mathrm{c}, i}=V_{\mathrm{c}, 30.2 \mathrm{~kg}} \times\left(\mathrm{WT}_{i} / 30.2\right)^{\mathrm{k} 2}$ & $k 2$ & $1.88(17 \%)$ & $2.15(1.43-3.30)$ \\
Peripheral volume & $\left(\mathrm{V}_{\mathrm{p}} / \mathrm{F}\right)_{30.2 \mathrm{~kg}}(\mathrm{~L})$ & $255(14 \%)$ & $252(197-338)$ \\
$V_{\mathrm{p}, i}=V_{\mathrm{p}, 30.2 \mathrm{~kg}} \times\left(\mathrm{WT}_{i} / 30.2\right)^{\mathrm{k} 3}$ & $k 3$ & $0.91(23 \%)$ & $0.88(0.60-1.21)$ \\
Intercompartmental clearance $^{\dagger}$ & $(\mathrm{Q} / \mathrm{F})_{30.2 \mathrm{~kg}}(\mathrm{~L} / \mathrm{h})$ & $121.8(21 \%)$ & $115.6(73.7-163)$ \\
$Q_{i}=Q_{30.2 \mathrm{~kg}} \times\left(\mathrm{WT}_{i} / 30.2\right)^{\mathrm{k} 4}$ & $k 4$ & $0.75 \mathrm{fix}$ & 0.75 fix \\
Absorption rate constant & $k_{\mathrm{a}}\left(\mathrm{h}{ }^{-1}\right)$ & $3.5 \mathrm{fix}$ & $3.5 \mathrm{fix}$ \\
IIV clearance & $\omega^{2} \mathrm{CL} / \mathrm{F}$ & $0.158(41 \%)$ & $0.145(0.063-0.259)$ \\
IIV volume of distribution & $\omega^{2} V_{\mathrm{c}} / \mathrm{F}$ & $1.19(27 \%)$ & $1.06(0.64-1.71)$ \\
Proportional error & $\sigma^{2}$ & $0.283(13 \%)$ & $0.272(0.222-0.346)$ \\
\hline
\end{tabular}

RSE is the relative standard error, and $90 \mathrm{CI}$ is the $90 \%$ confidence interval representing the 5th and 95th percentiles. Interindividual and residual variability values are shown as variance estimates

${ }^{\dagger}$ Parameters are apparent parameters, as only oral data was included 
what age the clearance of the selected substrates can at least be extrapolated from adult values with the covariate relationships for midazolam clearance, based on the differences in extraction ratio and $f_{\mathrm{u}}$ according to the framework that was previously reported (7). Based on this information, Fig. 1 was derived showing when scaling of pediatric clearance of a CYP3A substrate will be accurate depending on its extraction ratio and $f_{\mathrm{u}}$ values in adults. This figure shows that this method will accurately scale pediatric clearance values down to neonates of 1 day of age for alprazolam, atorvastatin, quinidine, sildenafil, solifenacin, sufentanil, and tacrolimus, while for the other drugs clearance will be at least accurately scaled down to infants of 1 month (sirolimus) and 6 months of age (cisapride, domperidone, and vincristine). Tamsulosin clearance scaling will be accurate down to at least 2 years of age, while for simvastatin accurate scaling down to 5 years of age may not even be possible (Fig. 1).

From Fig. 1 it can also be derived that a pediatric covariate function for midazolam can be used to scale CYP3A-mediated clearance across all ages including neonates (i.e., green bars) of HSA-bound substrates which are highly protein bound $\left(>90 \%, f_{\mathrm{u}} \leq 0.1\right)$, provided the extraction ratio in adults ranges between 0.05 and 0.55 . Similarly, for HSA-bound substrates with low protein binding $(<10 \%$, $\left.f_{\mathrm{u}} \geq 0.9\right)$, the drug to which the covariate function can be extrapolated should have an extraction ratio between 0.35 and 0.65 . In between these extreme percentages of binding to HSA, the required extraction ratio gradually changes between these values (green bars, Fig. 1). For AAG-bound drugs, fewer combinations of drug properties lead to accurate scaling based on a midazolam pediatric covariate function, with no scenarios for drugs with low or intermediate protein binding $\left(<60 \%, f_{\mathrm{u}} \geq 0.4\right)$, while an extraction ratio of $0.4-0.6$ or $0.1-0.5$ in adults leads to accurate scaling for drugs that are around $90 \%$ or $\geq 97.5 \%$ bound, respectively (Fig. 1).

a

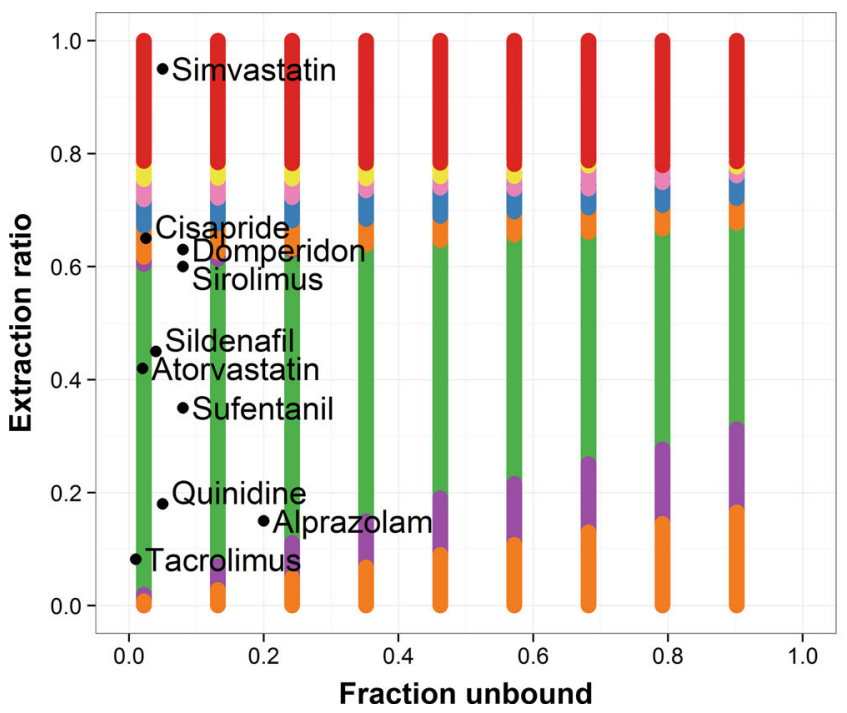

Comparison of Scaled Versus Reported Pediatric Clearance Values

Obtained pediatric and adult clearance values of CYP3A substrates are summarized in Table SI $(32,35,55-65)$. In Fig. 2, the scaled clearance values are shown together with the reported pediatric clearance values for the various substrates versus body weight. Table IV lists the calculated prediction errors for the three typical pediatric individuals. For most drugs, the scaled covariate relationships fall within the range of observed values, except for vincristine and sirolimus. The calculated PE values also show that scaled vincristine and sirolimus clearance values are inaccurate; although with a PE value of $64.3 \%$ and $58.3 \%$, respectively, this inaccuracy is not extreme. The PE values for all other drugs are $<50 \%$, indicating accurate or reasonably accurate scaling of clearance in infants, children, and adolescents.

\section{Sildenafil Population PK Models}

The reference model and extrapolation model for sildenafil described the sildenafil concentrations with a onecompartmental model. Table III presents model parameters and bootstrap values for both models and the goodness-of-fit plots and results from the NPDE analyses are presented in fig. S4 and fig. S5, respectively. These results show that descriptive and predictive properties of both models are similar.

In the reference model, apparent sildenafil clearance for a typical individual of $28 \mathrm{~kg}$ was found to be $41.9 \mathrm{~L} / \mathrm{h}$, and clearance increased exponentially with increasing body weight (exponent of 1.08 [RSE $11 \%]$ ), leading to an apparent clearance of $113 \mathrm{~L} / \mathrm{h}$ for a $70-\mathrm{kg}$ individual. In the extrapolation model, apparent clearance was scaled using Eq. 5, with a $\mathrm{CL}_{\text {adult }}$ of $100 \mathrm{~L} / \mathrm{h}$ for a $70-\mathrm{kg}$

b

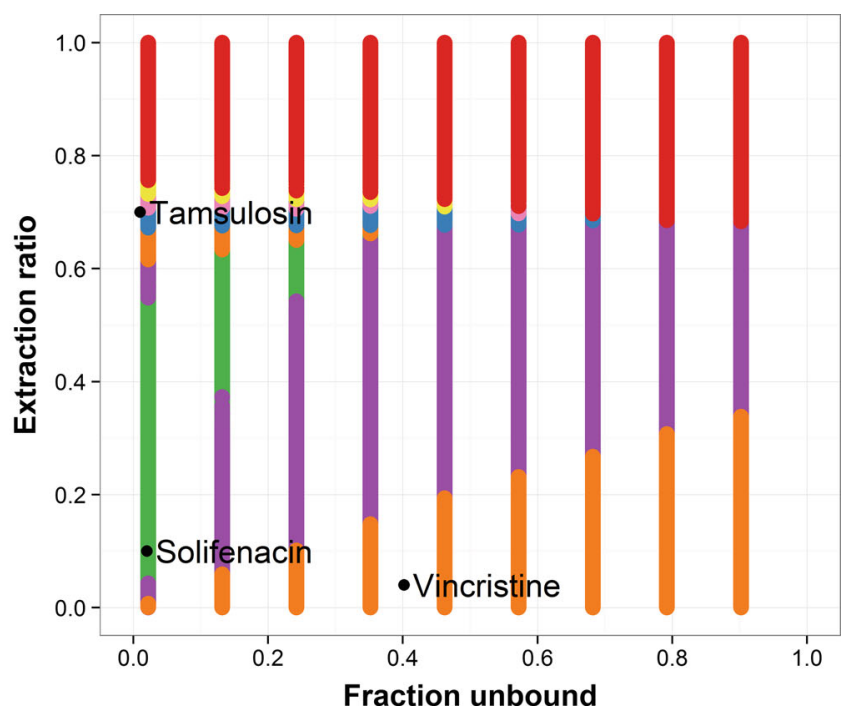

Fig. 1. Prediction of the age down to which the pediatric covariate function for CYP3A-mediated midazolam clearance can be used to accurately scale clearance of CYP3A substrates with specific drug properties. Extraction ratio values of the tested CYP3A substrates are plotted versus the fraction unbound $\left(f_{\mathrm{u}}\right)$ of the test drugs in adults. The color scheme was obtained from the published framework (7) and represents hypothetical model-test drug combinations that lead to systematically accurate scaling of clearance in children down to 1 day (green), 1 month (purple), 6 months (orange), 1 year (blue), 2 years (pink), and 5 years of age (yellow). Red indicates that scaling is not systematically accurate in children of 5 years and younger. The black data points represent the included test drugs and their extraction ratio and $f_{\mathrm{u}}$ values. Panel a shows drugs binding to albumin (HSA), while panel $\mathrm{b}$ shows drugs binding to $\alpha 1$-acid glycoprotein (AAG). Modified from Calvier et al. (7) (with permission) 

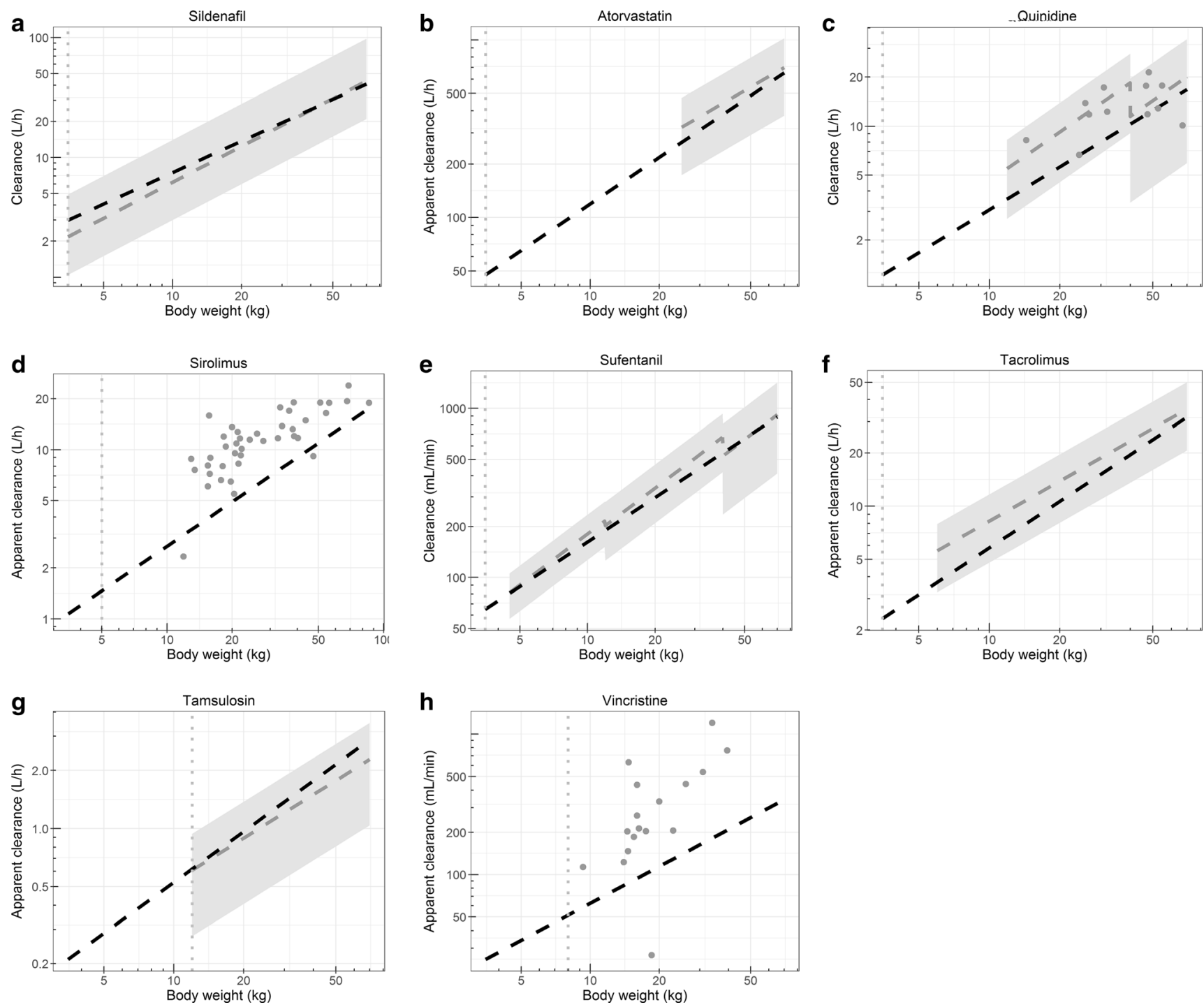

Fig. 2. Scaled and reported clearance values versus body weight for various CYP3A substrates. Clearance (or apparent clearance) values are based on the between-drug extrapolation of the pediatric covariate function for CYP3A-mediated midazolam clearance and reported adult clearance values (black) and based on reported pediatric clearance values in literature (gray), for sildenafil (a), atorvastatin (b), quinidine (c), sirolimus (d), sufentanil (e), tacrolimus (f), tamsulosin (g), and vincristine (h). The vertical dotted line (gray) indicates the body weight down to which systemically accurate clearance scaling is predicted to be possible according to the framework (7). For the reported clearance values the following is depicted: a Mean sildenafil clearance (line) with minimal and maximal reported values (gray area). b Typical atorvastatin clearance (line) $\pm 46.3 \%$ (\% CV, gray area). c Mean quinidine clearance (line) \pm 2 SD (gray area) and individual-reported clearances (closed circles). d Individual-reported sirolimus clearances (closed circles). e Mean sufentanil clearance (line) \pm 2 SD (gray area). f Typical tacrolimus clearance (line) $\pm 41.6 \%\left(\% \mathrm{CV}\right.$, with $\mathrm{CV}=\sqrt{e^{\sigma^{2}}-1}$, and $\sigma^{2}=0.16$, gray area). g Typical tamsulosin clearance (line) $\pm 54.4 \%(\% \mathrm{CV}$, gray area). h Individual-reported vincristine clearances, corrected for body surface area (closed circles)

individual, leading to a scaled apparent clearance of $44.9 \mathrm{~L} / \mathrm{h}$ for a 28-kg individual. As shown in a graphical comparison in Fig. 3a, both covariate relationships are very similar, with only a small difference in clearance values between the two models for children with the lowest body weight. Figure $3 \mathrm{~b}$ shows that when individual clearance predictions by both models are plotted versus age, the loess function for these relationships is also similar with the small difference in the youngest age range. These small discrepancies may be due to the small number of individuals in the youngest age group (1-2 years of age) in the population receiving midazolam used for establishing the pediatric covariate function.

The PE for clearance increases with a decreasing age, with a PE of $-5.2 \%, 14.6 \%$, and $32.1 \%$, in an adolescent, child, and infant, respectively, indicating that with a decreasing age and body weight, the extrapolation model yields a larger overprediction of clearance. However, the scaled clearance values are within the range of observed clearance values, which show a high variability throughout the pediatric age range (Fig. 3b).

\section{DISCUSSION}

Accurate scaling of plasma clearance is essential to establish optimal first-in-child doses during drug development and for the development of pediatric dose recommendations. As many drugs are metabolized by CYP3A enzymes and midazolam is a commonly accepted probe drug for CYP3A, we aimed to evaluate 
Table III. Model Parameter Estimates for the Sildenafil "Reference Model" Versus the Sildenafil "Extrapolation Model" and the Bootstrap Results for both Models Based on $n=250$ Resampling

\begin{tabular}{|c|c|c|c|c|c|}
\hline \multirow[t]{2}{*}{ Parameter } & & \multicolumn{2}{|l|}{ Reference model } & \multicolumn{2}{|l|}{ Extrapolation model } \\
\hline & & Model estimate (RSE) & Bootstrap (90 CI) & Model estimate (RSE) & Bootstrap (90 CI) \\
\hline Sildenafil clearance $^{\dagger}$ & $(\mathrm{CL} / \mathrm{F})_{70 \mathrm{~kg}}(\mathrm{~L} / \mathrm{h})$ & $113(13 \%)$ & $112(84.6-149)$ & $100 \mathrm{fix}$ & $100 \mathrm{fix}$ \\
\hline $\mathrm{CL}_{i}=\mathrm{CL}_{70 \mathrm{~kg}} \times\left(\mathrm{WT}_{i} / 70\right)^{\mathrm{k} 1}$ & $k 1$ & $1.08(11 \%)$ & $1.05(0.82-1.30)$ & 0.874 fix & 0.874 fix \\
\hline Volume of distribution ${ }^{\dagger}$ & $(\mathrm{V} / \mathrm{F})_{28 \mathrm{~kg}}(\mathrm{~L})$ & $540(33 \%)$ & $561(311-1424)$ & $590(29 \%)$ & $574(389-1134)$ \\
\hline$V_{i}=V_{28 \mathrm{~kg}} \times\left(\mathrm{WT}_{i} / 28\right)^{\mathrm{k} 2}$ & $k 2$ & $3.18(10 \%)$ & $3.17(2.41-4.27)$ & $3.18(10 \%)$ & $3.16(2.49-4.01)$ \\
\hline Absorption rate constant & $k_{\mathrm{a}}\left(\mathrm{h}^{-1}\right)$ & 1 fix & 1 fix & 1 fix & 1 fix \\
\hline IIV clearance & $\omega^{2} \mathrm{CL} / \mathrm{F}$ & $0.493(14 \%)$ & $0.487(0.363-0.631)$ & $0.510(13 \%)$ & $0.512(0.397-0.650)$ \\
\hline Proportional error & $\sigma^{2}$ & $0.627(7 \%)$ & $0.616(0.538-0.703)$ & $0.651(8 \%)$ & $0.646(0.564-0.738)$ \\
\hline
\end{tabular}

RSE is the relative standard error, and $90 \mathrm{CI}$ is the $90 \%$ confidence interval representing the 5 th and 95 th percentiles. Interindividual and residual variability values are shown as variance estimates

${ }^{\dagger}$ Parameters are apparent parameters, as only oral data was included

when a pediatric covariate function for CYP3A-mediated midazolam clearance can be used to scale pediatric clearance of CYP3A substrates given the recently reported guidance on between-drug extrapolation of covariate models.

Whether in this case, scaling of pediatric clearance of CYP3A substrates based on a covariate function from a pediatric population PK model for midazolam is accurate may depend on the drug properties as was reported by Calvier et al. before (7). We used this previously developed framework (7) to assess for which of the selected CYP3A substrates scaling with the pediatric covariate function from midazolam will lead to accurate clearance values (Fig. 1). The color code in Fig. 1 indicates down to which age scaling of clearance is expected to be systemically accurate based on the extraction ratio and $f_{\mathrm{u}}$ in adults. Each colored dot in this graph represents multiple drugs with differences in the remaining drug properties (i.e., blood-to-plasma partitioning and affinity to isoenzymes) and it should be noted that when the framework predicts that scaling of clearance is not systemically accurate for all drugs with the indicated combination of drug properties, there may still be drugs within the set of drugs represented by a data point for which this scaling is accurate. In those cases, it can however not be predicted a priori whether this will be the case for each of the individual drugs (7).

For the selected CYP3A substrates alprazolam, atorvastatin, cisapride, domperidone, quinidine, sildenafil, solifenacin, sufentanil, sirolimus, tacrolimus, and vincristine, based on differences in $f_{\mathrm{u}}$ and extraction ratio in comparison with midazolam, scaling of clearance with the covariate function of midazolam is expected to be accurate down to children of at least 1 year of age and for some drugs even to neonates and infants (Fig. 1).

Several approaches and methods for scaling of clearance in children have been described in literature, including scaling of clearance using a body weight-based exponential function with exponents of, e.g., $0.67,0.75$, or 1 . While some studies showed that allometric scaling may be a reasonable approach $(66,67)$ and other studies disagreed $(68,69)$, in a systematic assessment of the applicability of body weight-based scaling with a fixed exponent of 0.75 , it was found that this approach leads to increasingly inaccurate scaled values with decreasing age, reaching prediction errors of up to $278 \%$ in neonates (70). Also, other phenotyping studies have used probe drugs
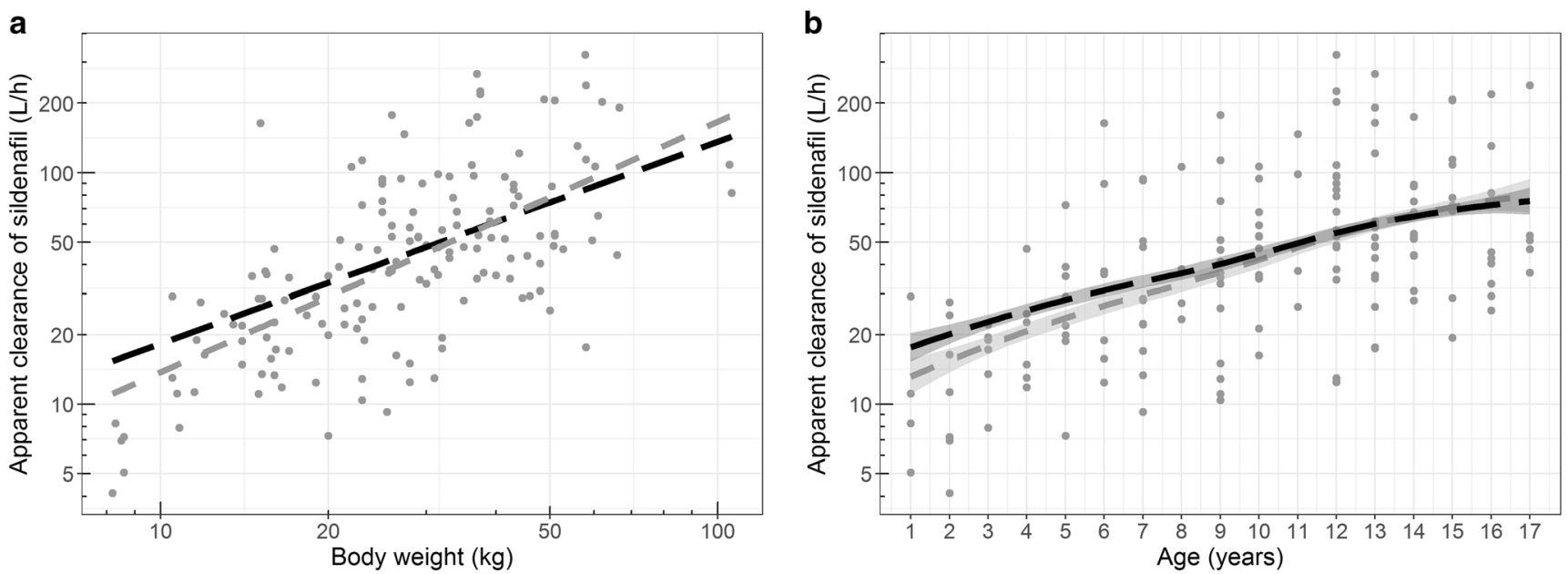

Fig. 3. Sildenafil apparent clearance versus body weight (a) and versus age (b) for the sildenafil reference model (gray) and based on betweendrug extrapolation of clearance (black) with points representing the individual-predicted clearance by the reference model. In panel a, the lines represent population-predicted clearance values directly derived from the body weight-based covariate relationship, while in panel $b$, the lines represent the loess function summarizing the population-predicted clearance values with a $95 \%$ confidence interval (shaded area) 
to predict clearance of a drug sharing its elimination pathway (71-74). These phenotyping studies include studies applying the "cocktail" approach with five drugs reflecting clearance by five CYP enzymes including CYP3A, which has been considered to be predictive of drug-drug interactions with regard to these enzymes (75), but there is no data on how this approach may predict pediatric clearance. The concept of using probe drugs for drug clearance in the pediatric population by extrapolating pediatric covariate functions for clearance for drugs sharing elimination pathways was therefore developed, and this method had already been successful in scaling pediatric clearance for UGT2B7 substrates and for drugs eliminated through glomerular filtration (4-6). Later, a systematic assessment of this method defined the prerequisites for systematically accurate scaling with this technique (7). In the current work, we illustrate how the knowledge obtained in that analysis can be applied. Moreover, we add CYP3A metabolism to the list of elimination pathways for which between-drug extrapolation of pediatric covariate relationships for clearance has been successfully applied.

Between-drug extrapolation of clearance on the basis of a pediatric covariate function for CYP3A-mediated midazolam clearance, indeed, led to accurate or reasonably accurate scaling of pediatric clearance of most of the selected CYP3A substrates in children (Table IV, Fig. 2). The pediatric covariate function for midazolam clearance can accurately scale pediatric clearance of CYP3A substrates down to at least 1 year of age for a large number of relevant substrates including sildenafil, atorvastatin, quinidine, sufentanil, tacrolimus, and tamsulosin. This indicates accurate predictions for $75 \%$ (6 out of 8 ) of the evaluated compounds. In addition to reported clearance values, for sildenafil, concentrationtime data were available as well. With these data, it was further confirmed that the between-drug extrapolation of the covariate relationship of midazolam clearance yields accurate clearance predictions.

Contrary to what was expected based on the theoretical framework, scaled clearance values of sirolimus and vincristine (2 out of 8 evaluated compounds) were inaccurate compared with

Table IV. Prediction error (PE) of scaled clearance values using the pediatric covariate function for CYP3A-mediated midazolam clearance versus reported pediatric clearance values for three representative pediatric subjects of 10,20, and $50 \mathrm{~kg}$ (Eq. 4), with negative and positive values for under- and overpredicted clearance values, respectively

\begin{tabular}{llll}
\hline & Infant $(10 \mathrm{~kg})$ & Child $(20 \mathrm{~kg})$ & Adolescent $(50 \mathrm{~kg})$ \\
\hline Atorvastatin & $-\mathbf{2 6 . 7} \%$ & $-\mathbf{2 0 . 1} \%$ & $-\mathbf{1 0 . 5} \%$ \\
Quinidine & $-\mathbf{3 3 . 5} \%$ & $-\mathbf{3 9 . 0} \%$ & $-\mathbf{1 2 . 8} \%$ \\
Sildenafil & $\mathbf{2 0 . 7} \%$ & $\mathbf{1 0 . 6} \%$ & $-\mathbf{1 . 4} \%$ \\
Sirolimus & $\mathrm{NA}$ & $-58.3 \%$ & $-\mathbf{3 1 . 5} \%$ \\
Sufentanil & $-\mathbf{1 0 . 3} \%$ & $-\mathbf{1 2 . 0} \%$ & $\mathbf{1 . 1} \%$ \\
Tacrolimus & $-\mathbf{4 4 . 6} \%$ & $-\mathbf{3 9 . 6} \%$ & $-\mathbf{3 2 . 3} \%$ \\
Tamsulosin & $\mathrm{NA}$ & $\mathbf{8 . 1} \%$ & $\mathbf{2 1 . 2} \%$ \\
Vincristine & $-64.3 \%$ & $\mathrm{NA}$ & NA \\
\hline
\end{tabular}

NA denotes no pediatric or adult clearance values reported in literature

Colors indicate an accurate prediction (absolute $\mathrm{PE}<30 \%$, bold), a reasonably accurate prediction (absolute PE 30-50\%, bold italics), and an inaccurate prediction (absolute $\mathrm{PE} \geq 50 \%$, italics) reported literature values ( $\mathrm{PE}>50 \%$, Table IV). For sirolimus, this may be due to the known induction of hepatic CYP3A activity and possibly altered hepatic P-glycoprotein expression (76). The impact of hepatic transporters was not taken into consideration in the framework by Calvier et al., because the impact of these transporters on clearance and their maturation patterns in children remains largely unknown. The scaling of vincristine may be inaccurate, because it is predominantly metabolized by CYP3A5 (77), with a relative smaller contribution of CYP3A4-mediated metabolism compared with midazolam, while midazolam is mainly metabolized by CYP3A4 (12). No pharmacogenomics data on CYP3A polymorphisms were collected, which could have explained some of the observed interindividual variability in clearance. Other factors that may affect the accuracy of our pathway-specific scaling approach, apart from the hepatic extraction ratio and $f_{\mathrm{u}}$ in adults, include that the fraction eliminated by a certain pathway may be different from the $\geq 75 \%$ assumed here. It has for instance been shown that the age down to which clearance can accurately be scaled increases when the contribution of CYP3A metabolism to the overall hepatic metabolism is decreasing (7). Additionally, the contribution of minor elimination pathways to overall drug clearance has been ignored in the current analysis. Moreover, scenarios for the between-drug extrapolation of pediatric covariate functions for clearance of HSAbound drugs to AAG-bound drugs have not been investigated; therefore, we assumed midazolam to be AAG-bound when using its covariate function to scale the clearance of AAG-bound CYP3A substrates. The impact of this assumption would be largest in neonates and in the youngest children $<1$ year of age; as in these age groups, the concentration of AAG is known to vary more with age than the concentration of HSA, due to the fact that AAG concentrations take longer time to mature and reach adult levels (78). It should also be taken into account that stress and disease state may impact protein binding and thereby alter the unbound fraction within an individual over time $(79,80)$. Lastly, as in the sildenafil PK study, not many samples were taken shortly after administration; absorption rate constants for sildenafil could not be estimated and were therefore fixed at $1 \mathrm{~h}^{-1}$, which is between the reported values for $k_{\mathrm{a}}$ of $0.34 \mathrm{~h}^{-1}(81)$ and $4.51 \mathrm{~h}^{-1}(82)$. A sensitivity analysis showed that fixing it at different values had no impact on the scaled clearance values.

In this analysis, we only included midazolam PK data from children $>1$ year of age, and therefore, the pediatric covariate function for midazolam clearance we developed in this analysis cannot be used to scale clearance values of CYP3A substrates in neonates and infants $<1$ year of age. Extrapolation of the covariate relationship to (preterm) neonates and infants is anticipated to yield overprediction of clearance, as CYP3Amediated metabolism in this young age group is lower due to the large impact of maturation in the first weeks and months of life (83). To be able to apply this covariate function to scale CYP3Amediated clearance in neonates and young infants up to 1 year of age, the model should be extended with a covariate relationship for clearance based on data from children $<1$ year of age.

\section{CONCLUSION}

This analysis showed that a pediatric covariate relationship describing how midazolam clearance changes throughout the pediatric age range can be used to scale adult clearance values for many other CYP3A substrates to pediatric clearance values. Specifically, it was found that this approach is applicable to 
accurately scale clearances of drugs that are mainly eliminated by CYP3A-mediated metabolism with for example high protein binding to HSA ( $>90 \%$ ) and a low to intermediate extraction ratio of $<0.55$ in adults. The possibility to scale clearance of CYP3A substrates with the appropriate properties in children from adult clearance using a pediatric covariate function for CYP3A-mediated midazolam clearance may improve pediatric dosing guidelines of CYP3A substrates for clinical practice and may aid in determining the dose in first-in-child studies involving new CYP3A substrates depending on its drug properties. This may be especially useful for CYP3A substrates in which scarce or no pediatric $\mathrm{PK}$ information is available, for example for alprazolam, domperidone, and solifenacin.

\section{ACKNOWLEDGMENTS}

The authors would like to thank Pfizer Inc. for kindly sharing their data on sildenafil PK in children of 1-16 years of age. Drs. Jeff Galinken and Peter Adamson were the PIs on the original midazolam PK study conducted at CHOP, and the study was supported by NIH/NICHD, Pediatric Pharmacology Research Unit, grant no. HD037255-06. The authors would like to thank Sebastiaan C. Goulooze (LACDR, Leiden University) for reviewing all scripts involved in this analysis. CAJ Knibbe is supported by a NWO Vidi grant (Vidi Knibbe, June 2013).

Open Access This article is distributed under the terms of the Creative Commons Attribution 4.0 International License (http://creativecommons.org/licenses/by/4.0/), which permits unrestricted use, distribution, and reproduction in any medium, provided you give appropriate credit to the original author(s) and the source, provide a link to the Creative Commons license, and indicate if changes were made.

\section{REFERENCES}

1. EMA. EMA (EMA/CHMP/EWP/147013/2004 Committee for Medicinal Products for Human Use [CHMP]). Guideline on the role of pharmacokinetics in the development of medicinal products in the paediatric population. 2007. www.ema.europa.eu/documents/scientific-guideline/guideline-role-pharmacokinetics-development-medicinal-products-paediatric-population_en.pdf. Accessed 18 Dec 2017.

2. FDA. General Clinical pharmacology considerations for pediatric studies for drugs and biological products, guidance for industry, 2014. Available from: https://www.fda.gov/downloads/ Drugs/GuidanceComplianceRegulatoryInformation/Guidances/ UCM425885.pdf.Accessed December 2017

3. Zisowsky J, Krause A, Dingemanse J. Drug development for pediatric populations: regulatory aspects. Pharmaceutics. 2010;2(4):364-88.

4. Krekels EH, Neely M, Panoilia E, Tibboel D, Capparelli E, Danhof $M$, et al. From pediatric covariate model to semiphysiological function for maturation: part I-extrapolation of a covariate model from morphine to zidovudine. CPT Pharmacometrics Syst Pharmacol. 2012;1:e9.

5. Zhao W, Biran V, Jacqz-Aigrain E. Amikacin maturation model as a marker of renal maturation to predict glomerular filtration rate and vancomycin clearance in neonates. Clin Pharmacokinet. 2013;52(12):1127-34.

6. De Cock RF, Allegaert K, Sherwin CM, Nielsen EI, de Hoog $\mathrm{M}$, van den Anker JN, et al. A neonatal amikacin covariate model can be used to predict ontogeny of other drugs eliminated through glomerular filtration in neonates. Pharm Res. 2014;31(3):754-67.

7. Calvier EAM, Krekels EHJ, Yu H, Valitalo PAJ, Johnson TN, Rostami-Hodjegan A, et al. Drugs being eliminated via the same pathway will not always require similar pediatric dose adjustments. CPT Pharmacometrics Syst Pharmacol. 2018 Mar;7(3):175-185.

8. Michaels S, Wang MZ. The revised human liver cytochrome P450 "Pie": absolute protein quantification of CYP4F and CYP3A enzymes using targeted quantitative proteomics. Drug Metab Dispos. 2014;42(8):1241-51.

9. Ince I, Knibbe CA, Danhof M, de Wildt SN. Developmental changes in the expression and function of cytochrome P450 3A isoforms: evidence from in vitro and in vivo investigations. Clin Pharmacokinet. 2013;52(5):333-45.

10. Gorski JC, Hall SD, Jones DR, VandenBranden M, Wrighton SA. Regioselective biotransformation of midazolam by members of the human cytochrome P450 3A (CYP3A) subfamily. Biochem Pharmacol. 1994;47(9):1643-53.

11. Thummel KE, Shen DD, Podoll TD, Kunze KL, Trager WF, Hartwell PS, et al. Use of midazolam as a human cytochrome P450 3A probe: I. In vitro-in vivo correlations in liver transplant patients. J Pharmacol Exp Ther. 1994;271(1):549-56.

12. Tseng E, Walsky RL, Luzietti RA Jr, Harris JJ, Kosa RE, Goosen TC, et al. Relative contributions of cytochrome CYP3A4 versus CYP3A5 for CYP3A-cleared drugs assessed in vitro using a CYP3A4-selective inactivator (CYP3cide). Drug Metab Dispos. 2014;42(7):1163-73.

13. Dundee JW, Collier PS, Carlisle RJ, Harper KW. Prolonged midazolam elimination half-life. $\mathrm{Br} \mathrm{J}$ Clin Pharmacol. 1986;21(4):425-9.

14. von Moltke LL, Greenblatt DJ, Harmatz JS, Shader RI. Alprazolam metabolism in vitro: studies of human, monkey, mouse, and rat liver microsomes. Pharmacology. 1993;47(4):268-76.

15. Jacobsen W, Kuhn B, Soldner A, Kirchner G, Sewing KF, Kollman PA, et al. Lactonization is the critical first step in the disposition of the 3-hydroxy-3-methylglutaryl-CoA reductase inhibitor atorvastatin. Drug Metab Dispos. 2000;28(11):1369-78.

16. Bedford TA, Rowbotham DJ. Cisapride. Drug interactions of clinical significance. Drug Saf. 1996;15(3):167-75.

17. Simard C, Michaud V, Gibbs B, Masse R, Lessard E, Turgeon J. Identification of the cytochrome P450 enzymes involved in the metabolism of domperidone. Xenobiotica. 2004;34(11-12):1013-23.

18. Guengerich FP, Muller-Enoch D, Blair IA. Oxidation of quinidine by human liver cytochrome P-450. Mol Pharmacol. 1986;30(3):287-95.

19. Walker DK, Ackland MJ, James GC, Muirhead GJ, Rance DJ, Wastall $\mathrm{P}$, et al. Pharmacokinetics and metabolism of sildenafil in mouse, rat, rabbit, dog and man. Xenobiotica. 1999;29(3):297-310.

20. Prueksaritanont T, Gorham LM, Ma B, Liu L, Yu X, Zhao JJ, et al. In vitro metabolism of simvastatin in humans [SBT]identification of metabolizing enzymes and effect of the drug on hepatic P450s. Drug Metab Dispos. 1997;25(10):1191-9.

21. Swart PJ, Krauwinkel WJ, Smulders RA, Smith NN. Pharmacokinetic effect of ketoconazole on solifenacin in healthy volunteers. Basic Clin Pharmacol Toxicol. 2006;99(1):33-6.

22. Tateishi T, Krivoruk Y, Ueng YF, Wood AJ, Guengerich FP, Wood M. Identification of human liver cytochrome P-450 3A4 as the enzyme responsible for fentanyl and sufentanil Ndealkylation. Anesth Analg. 1996;82(1):167-72.

23. Sattler M, Guengerich FP, Yun CH, Christians U, Sewing KF. Cytochrome P-450 3A enzymes are responsible for biotransformation of FK506 and rapamycin in man and rat. Drug Metab Dispos. 1992;20(5):753-61.

24. Vincent SH, Karanam BV, Painter SK, Chiu SH. In vitro metabolism of FK-506 in rat, rabbit, and human liver microsomes: identification of a major metabolite and of cytochrome $\mathrm{P} 4503 \mathrm{~A}$ as the major enzymes responsible for its metabolism. Arch Biochem Biophys. 1992;294(2):454-60.

25. Kamimura H, Oishi S, Matsushima H, Watanabe T, Higuchi S, Hall $\mathrm{M}$, et al. Identification of cytochrome P450 isozymes involved in metabolism of the alpha1-adrenoceptor blocker 
tamsulosin in human liver microsomes. Xenobiotica. 1998;28(10):909-22.

26. Zhou XJ, Zhou-Pan XR, Gauthier T, Placidi M, Maurel P, Rahmani R. Human liver microsomal cytochrome P450 3A isozymes mediated vindesine biotransformation. Metab Drug Inter Biochem Pharmacol. 1993;45(4):853-61.

27. Barst RJ, Ivy DD, Gaitan G, Szatmari A, Rudzinski A, Garcia $\mathrm{AE}$, et al. A randomized, double-blind, placebo-controlled, dose-ranging study of oral sildenafil citrate in treatment-naive children with pulmonary arterial hypertension. Circulation. 2012;125(2):324-34.

28. Gupta M EA, Willmann S, Adamson PC, Galinkin JL, Barrett JS. Model-based approaches to investigate pharmacogenetic and developmental sources of variation in the pharmacokinetics of midazolam after oral administration in children. 2006 [Available from: AAPS 2006. Abstract 003255. https://abstracts.aaps.org/ Published/Browse.aspx. Accessed December 2017.

29. Reed MD, Rodarte A, Blumer JL, Khoo KC, Akbari B, Pou S, et al. The single-dose pharmacokinetics of midazolam and its primary metabolite in pediatric patients after oral and intravenous administration. J Clin Pharmacol. 2001;41(12):1359-69.

30. Comets E, Brendel K, Mentre F. Computing normalised prediction distribution errors to evaluate nonlinear mixedeffect models: the npde add-on package for R. Comput Methods Prog Biomed. 2008;90(2):154-66.

31. Pfizer. Study protocol "A randomized, double-blind, placebocontrolled study of sildenafil in children With pulmonary arterial hypertension". https://clinicaltrials.gov/ct2/show/NCT00159913. Accessed December 2017.

32. European Medicines Agency. Revatio product information. Available from: http://www.ema.europa.eu/docs/en_GB/ document_library/EPAR_-_Product_Information/human/ 000638/WC500055840.pdf. Accessed 18 December 2017.

33. Frechen S, Junge L, Saari TI, Suleiman AA, Rokitta D, Neuvonen PJ, et al. A semiphysiological population pharmacokinetic model for dynamic inhibition of liver and gut wall cytochrome $\mathrm{P} 4503 \mathrm{~A}$ by voriconazole. Clin Pharmacokinet. 2013;52(9):763-81.

34. Lown KS, Thummel KE, Benedict PE, Shen DD, Turgeon DK, Berent S, et al. The erythromycin breath test predicts the clearance of midazolam. Clin Pharmacol Ther. 1995;57(1):16-24.

35. DrugBank Canada. Available from https://www.drugbank.ca. Accessed 17 July 2017.

36. Lennernas H. Clinical pharmacokinetics of atorvastatin. Clin Pharmacokinet. 2003;42(13):1141-60.

37. CVZ. College voor zorgverzekeringen Nederland; Farmacotherapeutisch Kompas (Dutch Pharmacotherapeutic Compass) 2013 [updated April 1, 2013. http://www.fk.cvz.nl/]. Accessed December 2017.

38. Rampe D, Roy ML, Dennis A, Brown AM. A mechanism for the proarrhythmic effects of cisapride (Propulsid): high affinity blockade of the human cardiac potassium channel HERG. FEBS Lett. 1997;417(1):28-32.

39. Heykants J, Hendriks R, Meuldermans W, Michiels M, Scheygrond $\mathrm{H}$, Reyntjens $\mathrm{H}$. On the pharmacokinetics of domperidone in animals and man. IV. The pharmacokinetics of intravenous domperidone and its bioavailability in man following intramuscular, oral and rectal administration. Eur J Drug Metab Pharmacokinet. 1981;6(1):61-70.

40. Simcyp (R) simulator version 15.1. Certara, Sheffield, United Kingdom. Available from: https://www.certara.com/software/ physiologically-based-pharmacokinetic-modeling-and-simulation/simcyp-simulator/. Accessed December 2017.

41. Bressler R, Bahl JJ. Principles of drug therapy for the elderly patient. Mayo Clin Proc. 2003;78(12):1564-77.

42. Zhao P, Zhang L, Grillo JA, Liu Q, Bullock JM, Moon YJ, et al. Applications of physiologically based pharmacokinetic (PBPK) modeling and simulation during regulatory review. Clin Pharmacol Ther. 2011;89(2):259-67.

43. Mehrotra N, Gupta M, Kovar A, Meibohm B. The role of pharmacokinetics and pharmacodynamics in phosphodiesterase5 inhibitor therapy. Int J Impot Res. 2007;19(3):253-64.

44. Gruer PJ, Vega JM, Mercuri MF, Dobrinska MR, Tobert JA. Concomitant use of cytochrome P450 3A4 inhibitors and simvastatin. Am J Cardiol. 1999;84(7):811-5.
45. Benet LZ, Cummins CL, Wu CY. Unmasking the dynamic interplay between efflux transporters and metabolic enzymes. Int J Pharm. 2004;277(1-2):3-9.

46. Osmulski PA, Gaczynska M. Rapamycin allosterically inhibits the proteasome. Mol Pharmacol. 2013;84(1):104-13.

47. Doroshyenko O, Fuhr U. Clinical pharmacokinetics and pharmacodynamics of solifenacin. Clin Pharmacokinet. 2009;48(5):281-302.

48. Raucoules-Aime M, Kaidomar M, Levron JC, Le Moing JP, Goubaux B, Gugenheim J, et al. Hepatic disposition of alfentanil and sufentanil in patients undergoing orthotopic liver transplantation. Anesth Analg. 1997;84(5):1019-24.

49. Saari TI, Ihmsen H, Mell J, Frohlich K, Fechner J, Schuttler J, et al. Influence of intensive care treatment on the protein binding of sufentanil and hydromorphone during pain therapy in postoperative cardiac surgery patients. $\mathrm{Br} \mathrm{J}$ Anaesth. 2014;113(4):677-87.

50. Tuteja S, Alloway RR, Johnson JA, Gaber AO. The effect of gut metabolism on tacrolimus bioavailability in renal transplant recipients. Transplantation. 2001;71(9):1303-7.

51. Piekoszewski W, Jusko WJ. Plasma protein binding of tacrolimus in humans. J Pharm Sci. 1993;82(3):340-1.

52. Matsushima H, Kamimura H, Soeishi Y, Watanabe T, Higuchi S, Tsunoo M. Pharmacokinetics and plasma protein binding of tamsulosin hydrochloride in rats, dogs, and humans. Drug Metab Dispos. 1998;26(3):240-5.

53. Dennison JB, Mohutsky MA, Barbuch RJ, Wrighton SA, Hall $\mathrm{SD}$. Apparent high CYP3A5 expression is required for significant metabolism of vincristine by human cryopreserved hepatocytes. J Pharmacol Exp Ther. 2008;327(1):248-57.

54. Chan JD. Pharmacokinetic drug interactions of vinca alkaloids: summary of case reports. Pharmacotherapy. 1998;18(6):1304-7.

55. Knebel W, Gastonguay MR, Malhotra B, El-Tahtawy A, Jen F, Gandelman K. Population pharmacokinetics of atorvastatin and its active metabolites in children and adolescents with heterozygous familial hypercholesterolemia: selective use of informative prior distributions from adults. J Clin Pharmacol. 2013;53(5):505-16.

56. Dansirikul C, Morris RG, Tett SE, Duffull SB. A Bayesian approach for population pharmacokinetic modelling of sirolimus. Br J Clin Pharmacol. 2006;62(4):420-34.

57. Scholz J, Steinfath M, Schulz M. Clinical pharmacokinetics of alfentanil, fentanyl and sufentanil. An update. Clin Pharmacokinet. 1996;31(4):275-92.

58. Saeves I, Line PD, Bremer S, Vethe NT, Tveit RG, Meltevik TJ, et al. Tacrolimus exposure and mycophenolate pharmacokinetics and pharmacodynamics early after liver transplantation. Ther Drug Monit. 2014;36(1):46-53.

59. Tsuda Y, Tatami S, Yamamura N, Tadayasu Y, Sarashina A, Liesenfeld KH, et al. Population pharmacokinetics of tamsulosin hydrochloride in paediatric patients with neuropathic and non-neuropathic bladder. $\mathrm{Br} \mathrm{J}$ Clin Pharmacol. 2010;70(1):88-101.

60. de Graaf SS, Bloemhof H, Vendrig DE, Uges DR. Vincristine disposition in children with acute lymphoblastic leukemia. Med Pediatr Oncol. 1995;24(4):235-40.

61. Hill KD, Sampson MR, Li JS, Tunks RD, Schulman SR, Cohen-Wolkowiez M. Pharmacokinetics of intravenous sildenafil in children with palliated single ventricle heart defects: effect of elevated hepatic pressures. Cardiol Young. 2016;26(2):354-62.

62. Szefler SJ, Pieroni DR, Gingell RL, Shen DD. Rapid elimination of quinidine in pediatric patients. Pediatrics. 1982;70(3):370-5.

63. Scott JR, Courter JD, Saldana SN, Widemann BC, Fisher M, Weiss B, et al. Population pharmacokinetics of sirolimus in pediatric patients with neurofibromatosis type 1 . Ther Drug Monit. 2013;35(3):332-7.

64. Greeley WJ, de Bruijn NP, Davis DP. Sufentanil pharmacokinetics in pediatric cardiovascular patients. Anesth Analg. 1987;66(11):1067-72.

65. Jalil MH, Hawwa AF, McKiernan PJ, Shields MD, McElnay JC. Population pharmacokinetic and pharmacogenetic analysis of tacrolimus in paediatric liver transplant patients. $\mathrm{Br} \mathrm{J}$ Clin Pharmacol. 2014;77(1):130-40. 
66. Holford N, Heo YA, Anderson B. A pharmacokinetic standard for babies and adults. J Pharm Sci. 2013;102(9):2941-52.

67. Liu T, Ghafoori P, Gobburu JV. Allometry is a reasonable choice in pediatric drug development. J Clin Pharmacol. 2017;57(4):469-75.

68. Strougo A, Eissing T, Yassen A, Willmann S, Danhof M, Freijer J. First dose in children: physiological insights into pharmacokinetic scaling approaches and their implications in paediatric drug development. J Pharmacokinet Pharmacodyn. 2012;39(2):195-203.

69. Mahmood I. Misconceptions and issues regarding allometric scaling during the drug development process. Expert Opin Drug Metab Toxicol. 2018;14(8):843-54.

70. Calvier EA, Krekels EH, Valitalo PA, Rostami-Hodjegan A, Tibboel D, Danhof M, et al. Allometric scaling of clearance in paediatric patients: when does the magic of 0.75 fade? Clin Pharmacokinet. 2017;56(3):273-85.

71. Parra-Guillen ZP, Berger PB, Haschke M, Donzelli M, Winogradova D, Pfister B, et al. Role of cytochrome P450 $3 \mathrm{~A} 4$ and $1 \mathrm{~A} 2$ phenotyping in patients with advanced non-smallcell lung cancer receiving erlotinib treatment. Basic Clin Pharmacol Toxicol. 2017;121(4):309-15.

72. Berger B, Bachmann F, Duthaler U, Krahenbuhl S, Haschke M. Cytochrome P450 enzymes involved in metoprolol metabolism and use of metoprolol as a CYP2D6 phenotyping probe drug. Front Pharmacol. 2018;9:774.

73. Janssen A, Verkleij CPM, van der Vlist A, Mathijssen RHJ, Bloemendal HJ, Ter Heine R. Towards better dose individualisation: metabolic phenotyping to predict cabazitaxel pharmacokinetics in men with prostate cancer. Br J Cancer. 2017;116(10):1312-7.

74. Keller GA, Gago MLF, Diez RA, Di Girolamo G. In vivo phenotyping methods: cytochrome $\mathrm{P} 450$ probes with emphasis on the cocktail approach. Curr Pharm Des. 2017;23(14):2035-49.

75. Turpault S, Brian W, Van Horn R, Santoni A, Poitiers F, Donazzolo Y, et al. Pharmacokinetic assessment of a five-probe cocktail for CYPs 1A2, 2C9, 2C19, 2D6 and 3A. Br J Clin Pharmacol. 2009;68(6):928-35.

76. Bai S, Stepkowski SM, Kahan BD, Brunner LJ. Metabolic interaction between cyclosporine and sirolimus. Transplantation. 2004;77(10):1507-12.

77. Dennison JB, Jones DR, Renbarger JL, Hall SD. Effect of CYP3A5 expression on vincristine metabolism with human liver microsomes. J Pharmacol Exp Ther. 2007;321(2):553-63.

78. McNamara PJ, Alcorn J. Protein binding predictions in infants. AAPS PharmSci. 2002;4(1):E4.

79. Tokita K, Burke JF, Yoshizaki H, Fischer S, Schmid K. The constancy of the alpha-1-acid glycoprotein variants of normal adults under conditions of severe stress. J Clin Invest. 1966;45(10):1624-30.

80. De Cock PA, Desmet S, De Jaeger A, Biarent D, Dhont E, Herck I, et al. Impact of vancomycin protein binding on target attainment in critically ill children: back to the drawing board? J Antimicrob Chemother. 2017;72(3):801-4.

81. Olguin HJ, Martinez HO, Perez CF, Mendiola BR, Espinosa LR, Pacheco JLC, et al. Pharmacokinetics of sildenafil in children with pulmonary arterial hypertension. World J Pediatr. 2017;13(6):588-92.

82. Watt S, Hayashi N, Harnisch L, Gao X. Population pharmacokinetics (PK) of sildenafil in paedatric and adult patients with pulmonary arterial hypertension (PAH). European Society of Cardiology. Available from: http://spo.escardio.org/eslides/ view.aspx? eevtid=40\&fp=P4506. Accessed 2 April 2019.

83. Brussee JM, Vet NJ, Krekels EHJ, Valkenburg AJ, JacqzAigrain E, van Gerven JMA, et al. Predicting CYP3A-mediated midazolam metabolism in critically ill neonates, infants, children, and adults with inflammation and organ failure. Br J Clin Pharmacol. 2018;84(2):358-368.

Publisher's Note Springer Nature remains neutral with regard to jurisdictional claims in published maps and institutional affiliations. 